\title{
Web-based tool for dynamic functional outcome after acute ischemic stroke and comparison with existing models
}

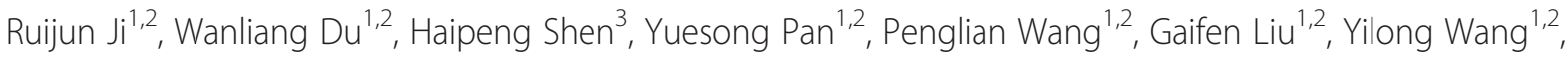 \\ Hao $\mathrm{Li}^{1,2}$, Xingquan Zhao ${ }^{1,2}$, Yongjun Wang ${ }^{1,2^{*}}$ and On behalf of China National Stroke Registry (CNSR) \\ investigators
}

\begin{abstract}
Background: Acute ischemic stroke (AIS) is one of the leading causes of death and adult disability worldwide. In the present study, we aimed to develop a web-based risk model for predicting dynamic functional status at discharge, 3-month, 6-month, and 1-year after acute ischemic stroke (Dynamic Functional Status after Acute Ischemic Stroke, DFS-AIS).

Methods: The DFS-AIS was developed based on the China National Stroke Registry (CNSR), in which eligible patients were randomly divided into derivation (60\%) and validation (40\%) cohorts. Good functional outcome was defined as modified Rankin Scale (mRS) score $\leq 2$ at discharge, 3-month, 6-month, and 1-year after AIS, respectively. Independent predictors of each outcome measure were obtained using multivariable logistic regression. The area under the receiver operating characteristic curve (AUROC) and plot of observed and predicted risk were used to assess model discrimination and calibration.

Results: A total of 12,026 patients were included and the median age was 67 (interquartile range: $57-75$ ). The proportion of patients with good functional outcome at discharge, 3-month, 6-month, and 1-year after AIS was $67.9 \%, 66.5 \%, 66.9 \%$ and $66.9 \%$, respectively. Age, gender, medical history of diabetes mellitus, stroke or transient ischemic attack, current smoking and atrial fibrillation, pre-stroke dependence, pre-stroke statins using, admission National Institutes of Health Stroke Scale score, admission blood glucose were identified as independent predictors of functional outcome at different time points after AIS. The DFS-AIS was developed from sets of predictors of mRS $\leq 2$ at different time points following AIS. The DFS-AIS demonstrated good discrimination in the derivation and validation cohorts (AUROC range: 0.837-0.845). Plots of observed versus predicted likelihood showed excellent calibration in the derivation and validation cohorts (all $r=0.99, \mathrm{P}<0.001$ ). When compared to 8 existing models, the DFS-AIS showed significantly better discrimination for good functional outcome and mortality at discharge, 3-month, 6-month, and 1-year after AIS (all P $<0.0001$ ).
\end{abstract}

Conclusion: The DFS-AIS is a valid risk model to predict functional outcome at discharge, 3-month, 6-month, and 1-year after AIS.

Keywords: Acute ischemic stroke, Prognosis, Risk model

\footnotetext{
* Correspondence: yongjunwang1962@gmail.com

'Tiantan Comprehensive Stroke Center, Beijing Tiantan Hospital, Capital

Medical University, No. 6 Tiantanxili, Beijing 100050, Dongcheng District,

China

${ }^{2}$ China National Clinical Research Center for Neurological Diseases

(NCRC-ND), Beijing, China

Full list of author information is available at the end of the article
} 


\section{Background}

Stroke is one of the leading causes of death and adult disability worldwide and acute ischemic stroke (AIS) accounts for about $85 \%$ of all stroke cases [1,2]. At time of admission, clinicians are frequently requested to estimate the likelihood of good functional outcome in post-stroke period. During the past decades, a number of prognostic models for AIS have been developed [3-18]. Except the IScore and ASTRAL score [15,17], majority of them have not been externally validated. Meanwhile, none of them is recommended by international stroke guidelines and widely used in routine clinical practice or clinical trials $[3,19]$. In addition, the majority of existing models are designed to provide prognostic information for a single time point after AIS, such as at discharge [13], 30-day [10], 3-month [4-7,9,17], 6-month [14], 9-month [16] or 1-year $[8,11]$ after onset. Although few recently proposed risk models could provide dynamic prognostic information at multiple time points after AIS $[15,18]$, they mainly focused on mortality instead of functional status. In real-world practice, patients, family members, clinicians and researchers usually concern about not only the likelihood of survival, but also the likelihood of survival with function recovery after AIS.

In the study, we aimed to develop and validate a risk model to provide dynamic prognostic information on functional status at multiple time points (discharge, 3-month, 6-month, and 1-year) after AIS (Dynamic Functional Status after Acute Ischemic Stroke, DFS-AIS). Furthermore, we compared discrimination of the DFS-AIS and existing AIS models with regard to both functional outcome and mortality at discharge, 3-month, 6-month, and 1-year after AIS.

\section{Methods}

\section{Study population}

The derivation and validation cohort originated from the largest stroke registry in China, the China National Stroke Registry (CNSR) [20], which was a nationwide, multicenter, and prospective registry of consecutive patients with acute cerebrovascular events from September 2007 to August 2008. Briefly, hospitals in China are classified into 3 Levels: I (community hospitals); II (hospitals that serve several communities); and III (central hospitals for a certain district or city). Level III hospital is usually an academic center and more medically advanced than level I and II hospitals. Totally, 242 potential sites including 114 Level III, 71 Level II, and 57 Level I hospitals, from both urban and rural area, were initially identified by soliciting application. The CNSR steering committee evaluated research capability and commitment to the registry of each hospital with preliminary survey. Finally, a total of 132 hospitals including 100 Level III and 32 Level II were selected, which cover 27 provinces and 4 municipalities across China. Trained research coordinators at each institute reviewed medical records daily to identify, consent and enroll consecutively eligible patients. To be eligible for this study, subjects had to meet the following criteria: (1) age 18 years or older; (2) hospitalized with a primary diagnosis of AIS according to the World Health Organization criteria [21]; (3) stroke confirmed by head computerized tomography $(\mathrm{CT})$ or brain magnetic resonance imaging (MRI); (4) direct admission to hospital from a physician's clinic or emergency department; (5) written informed consent from patients or their legal representatives. For the present study, patients who received thrombolysis $(\mathrm{n}=389$, 3.1\%) (Additional file 1: Table S1) were excluded since thrombolysis alters the natural history of disability after stroke [22]. However, they were included in sensitivity analysis. The scientific use of data registered in the CNSR was approved by the central institutional review board at Beijing Tiantan hospital and local ethical committees at each participating hospital (Additional file 1).

\section{Data collection and variables definition}

Standardized case report form (CRF) was used for data collection in the CNSR network. The relevant data was extracted from the medical records by trained research coordinators. Data from each CRF were manually checked for completeness, correct coding, and proper application of diagnostic algorithm by a research specialist from an independent contract research organization. In the study, the following candidate variables were analyzed: (1) demographics (age and gender); (2) stroke risk factors: hypertension (history of hypertension or anti-hypertensive medication use), diabetes mellitus (history of diabetes mellitus or anti-diabetic medication use), dyslipidemia (history of dyslipidemia or lipid-lowering medication use), atrial fibrillation (history of atrial fibrillation or documentation of atrial fibrillation on admission), coronary heart disease, history of stroke/TIA, current smoking, and excess alcohol consumption ( $\geq 2$ standard alcohol beverages per day); (3) pre-existing comorbidities: congestive heart failure, valvular heart disease, peripheral artery disease, chronic obstructive pulmonary disease (COPD), hepatic cirrhosis, peptic ulcer or previous gastrointestinal bleeding (GIB), renal failure, Alzheimer's disease/ dementia, and cancer; (4) pre-stroke dependence (modified Rankin Scale score $\geq 3$ ); (5) pre-admission antithrombotic medications: anticoagulant with warfarin (for atrial fibrillation) or anti-platelet medication (aspirin, clopidogrel, or extended release dipyridamole combined with aspirin); (6) pre-admission statins using; (7) transportation mode to hospital (dichotomized as by emergency medical system [EMS] or private transportation [such as by taxi or private car]); (8) Time from onset to hospital arrival (hours); (9) admission systolic and diastolic 
blood pressure (mmHg); (10) admission stroke severity based on National Institutes of Health Stroke Scale (NIHSS) score; (11) stroke subtypes according to the Oxfordshire Community Stroke Project (OCSP) criteria [23], where AIS was classified into partial anterior circulation infarct (PACI), total anterior circulation infarct (TACI), lacunar infarction (LACI), and posterior circulation infarct (POCI). We did not use TOAST subtype because it requires the results of investigations that usually are not available at the time of admission for many patients; (12) admission blood glucose (mmol/L).

\section{Functional outcome assessment}

The modified Rankin Scale (mRS) was used to assess functional outcome at different time points (discharge, 3-month, 6-month and 1-year) after AIS. Treating physician evaluated patients' functional status at discharge. A central follow-up for functional status at 3-month, 6-month and 1-year after onset was made by telephone interview by trained interviewers based on a standardized interview protocol. Good functional outcome was defined as $\mathrm{mRS} \leq 2$ at discharge, 3-month, 6-month, and 1 -year after AIS, respectively.

\section{Statistical analysis}

We aimed to derivate and validate a risk model to predict functional outcome at multiple time points (discharge, 3-month, 6-month, and 1-year) after AIS by using patient characteristics commonly available at presentation. In order to guarantee the accuracy of prediction, we separately developed 4 prognostic rules for each outcome measures. For the practicability of the model, we developed a web-based and user-friendly calculator, which can automatically provide prognostic information at different time points after AIS.

The eligible patients (an additional figure shows this in more detail [see Additional file 1: Figure S1]) in the CNSR were randomly divided into derivation (60\%) and validation (40\%) cohorts. Model building was performed exclusively in the derivation cohort $(n=7,215)$. In univariate analysis, Chi-square or Mann-Whitney test was used as appropriate. Multivariate logistic regression was used to determine independent predictors of good functional outcome $(\mathrm{mRS} \leq 2)$ at discharge, 3-month, 6-month and 1 -year after AIS, respectively. Candidate variables were those with biologically plausible link to good functional outcome after AIS on the basis of prior publication and those associated with good functional outcome after AIS on univariate analysis $(\mathrm{P} \leq 0.1)$. On multivariate analysis, backward stepwise method was used. To test for collinearity between covariates of the final multivariable model, the tolerance and variance inflation factor (VIF) of each covariate were calculated. The resulting DFS-AIS was then validated by assessing model discrimination and calibration in the validation cohort $(n=4,811)$ [24]. Discrimination was assessed by calculating the area under the receiver operating characteristic curve (AUROC). Calibration was assessed by performing the HosmerLemeshow goodness-of-fit test. Due to that the HosmerLemeshow test has been shown to be overly sensitive to trivial deviations from the ideal fit when the sample size is large [25], model calibration was also graphically depicted in the plot of observed versus predicted risk according to 10 deciles of predicted risk.

Furthermore, we compared discrimination of the DFSAIS and existing AIS models with regard to both functional outcome $(\mathrm{mRS} \leq 2)$ and mortality at discharge, 3-month, 6-month and 1-year after AIS in the overall cohort $(n=12,026)$. The primary criterion for selection of compared model was whether all elements required for the model were available in our dataset. Meanwhile, due to that a systematical review on prognostic models of acute stroke published in 2001 indicated that none of the existing prognostic models have been sufficiently well developed and validated to be useful in either clinical practice or research, we included only AIS risk models developed after 2001. Finally, 8 risk models (the Weimar's survival and functional model [9], König's survival and functional model [12], GWTG score [13], IScore [15], ASTRAL score [17], and PLAN score [18]) were selected. Discrimination of these models (AUROC) was compared using the test proposed by Delong et al. [26]. Sensitivity, specificity, positive predict value (PPV), and negative predictive value (NPV) was calculated at each model's maximum Youden Index. Missing data were coded as following: 25 patients $(0.2 \%)$ with missing data on medical history of current smoking, $110(0.9 \%)$ of excess alcohol consumption, 30 (0.2\%) of Alzheimer disease/dementia, 50 (0.4\%) of hepatic cirrhosis were coded as absence. Sixty-sever patients (0.6\%) with missing or unknown time from stroke onset to hospital arrival were coded as median.

All tests were 2-tailed and statistical significance was determined at $\alpha$ level of 0.05 . Regarding the number of comparisons done in comparing discrimination of prognostic models, we use more restricted criterion (0.001) to declare significant difference. Statistical analysis was performed using SAS 9.3 (SAS Institute, Cary, NC), SPSS 20.0 (SPSS Inc., Chicago, IL), and Medcalc software 12.3 (MedCalc, Ostend, Belgium).

\section{Results}

Patient characteristics

Patient characteristics of the derivation and validation cohorts are shown in Table 1. From September 2007 to August 2008, a total of 12,026 patients in the CNSR were eligible for the study and included in final analysis (an additional figure shows this in more detail [see 
Table 1 Patient characteristics

\begin{tabular}{|c|c|c|c|c|}
\hline & $\begin{array}{l}\text { Overall cohort } \\
(\mathrm{N}=12026)\end{array}$ & $\begin{array}{l}\text { Derivation cohort } \\
(\mathrm{N}=7215)\end{array}$ & $\begin{array}{l}\text { Validation cohort } \\
(\mathrm{N}=4811)\end{array}$ & $P$ value \\
\hline \multicolumn{5}{|l|}{ Demographics } \\
\hline Age, y, median (IQR) & $67(57-75)$ & $67(57-75)$ & $67(57-75)$ & 0.35 \\
\hline Gender (male), n (\%) & $7411(61.6)$ & $4461(61.8)$ & $2950(61.3)$ & 0.58 \\
\hline \multicolumn{5}{|l|}{ Stroke risk factors, n (\%) } \\
\hline Hypertension & $7703(64.1)$ & $4602(63.8)$ & $3101(64.5)$ & 0.46 \\
\hline Diabetes mellitus & $2615(21.7)$ & $1580(21.9)$ & $1035(21.9)$ & 0.62 \\
\hline Dyslipidemia & $1349(11.2)$ & $797(11.0)$ & $552(11.5)$ & 0.47 \\
\hline Atrial fibrillation & $870(7.2)$ & $543(7.5)$ & $327(6.8)$ & 0.13 \\
\hline Coronary artery disease & $1714(14.3)$ & $1063(14.7)$ & $651(13.5)$ & 0.07 \\
\hline History of stroke/TIA & 4113 (33.6) & $2403(33.3)$ & $1635(34.0)$ & 0.44 \\
\hline Current Smoking & $4750(39.5)$ & $2846(39.4)$ & 1904 (39.6) & 0.89 \\
\hline Excess alcohol consumption & $1844(15.3)$ & $1124(15.6)$ & $720(15.0)$ & 0.37 \\
\hline \multicolumn{5}{|l|}{ Pre-existing comorbidities, n (\%) } \\
\hline Congestive heart failure & $239(2.0)$ & $151(2.1)$ & $88(1.8)$ & 0.32 \\
\hline Valvular heart disease & $284(2.4)$ & $171(2.4)$ & $113(2.3)$ & 0.95 \\
\hline COPD & $138(1.1)$ & $80(1.1)$ & $58(1.2)$ & 0.66 \\
\hline Hepatic cirrhosis & $42(0.4)$ & $24(0.3)$ & $18(0.4)$ & 0.76 \\
\hline Peptic ulcer or previous GIB & $411(3.4)$ & $235(3.3)$ & $178(3.7)$ & 0.24 \\
\hline Alzheimer's disease/Dementia & $166(1.4)$ & $95(1.3)$ & $71(1.5)$ & 0.47 \\
\hline Cancer & $222(1.8)$ & $140(1.9)$ & $82(1.7)$ & 0.36 \\
\hline Pre-stroke dependence (mRS $\geq 3$ ), n (\%) & $1140(9.5)$ & $684(9.5)$ & $456(9.5)$ & 0.99 \\
\hline Pre-admission antithrombotic therapy, n (\%) & $2246(18.7)$ & $1345(18.6)$ & $901(18.7)$ & 0.91 \\
\hline Warfarin (for atrial fibrillation) & $272(2.3)$ & $170(2.4)$ & $102(2.1)$ & \\
\hline Antiplatelet using & $2208(16.7)$ & $1201(16.6)$ & $807(16.8)$ & \\
\hline Pre-admission statins using, n (\%) & $5175(43.0)$ & $3066(42.5)$ & $2109(43.8)$ & 0.15 \\
\hline Transport to hospital by EMS, n (\%) & $1826(15.2)$ & $1075(14.9)$ & $751(15.6)$ & 0.29 \\
\hline Time from onset to arrival (hours), median (IQR) & $24(7-64)$ & $24(7-64)$ & $24(7-65)$ & 0.83 \\
\hline Admission SBP (mm Hg), median (IQR) & $150(136-164)$ & $150(135-163)$ & $150(136-164)$ & 0.76 \\
\hline Admission DBP (mm Hg), median (IQR) & $89(80-96)$ & 89 (80-96) & $89(80-96)$ & 0.67 \\
\hline Admission NIHSS score, median (IQR) & $5(2-9)$ & $5(2-10)$ & $5(2-9)$ & 0.33 \\
\hline $\mathrm{NIHSS}=0-4$ & $6152(51.2)$ & $3686(51.1)$ & $2466(51.3)$ & 0.31 \\
\hline $\mathrm{NIHSS}=5-9$ & $3159(26.3)$ & $1873(26.0)$ & $1286(26.7)$ & \\
\hline $\mathrm{NIHSS}=10-14$ & $1312(10.9)$ & $783(10.9)$ & $529(11.0)$ & \\
\hline NIHSS $\geq 15$ & $1403(11.7)$ & $873(12.1)$ & $530(11.0)$ & \\
\hline OCSP subtypes, n (\%) & & & & 0.69 \\
\hline Partial anterior circulation infarct (PACl) & $6698(55.7)$ & $4025(55.8)$ & $2673(55.6)$ & \\
\hline Total anterior circulation infarct (TACl) & $1035(8.6)$ & $623(8.6)$ & $412(8.6)$ & \\
\hline Lacunar infarction (LACl) & $2252(18.7)$ & $1365(18.9)$ & $887(18.4)$ & \\
\hline Posterior circulation infarct (POCl) & $2009(17.1)$ & $1180(16.8)$ & $829(17.6)$ & \\
\hline Admission blood glucose (mmol/L), median (IQR) & $6.2(5.5-7.0)$ & $6.2(5.5-7.0)$ & $6.2(5.5-7.1)$ & 0.30 \\
\hline Length of hospital stay, median (IQR) & $14(10-20)$ & $14(10-20)$ & $14(11-20)$ & 0.55 \\
\hline \multicolumn{5}{|l|}{$\mathrm{mRS} \leq 2$ within 1 year after onset, $\mathrm{n}(\%)$} \\
\hline At discharge & $8160(67.9)$ & $4885(67.7)$ & $3275(68.1)$ & 0.68 \\
\hline At 3-month & $7994(66.5)$ & $4771(66.1)$ & $3223(67.0)$ & 0.33 \\
\hline
\end{tabular}


Table 1 Patient characteristics (Continued)

\begin{tabular}{|c|c|c|c|c|}
\hline At 6-month & 8050 (66.9) & 4806 (66.6) & $3244(67.4)$ & 0.35 \\
\hline At 12-mont & $8047(66.9)$ & $4817(66.8)$ & $3230(67.1)$ & 0.68 \\
\hline \multicolumn{5}{|c|}{ Mortality within 1 year after onset, n (\%) } \\
\hline At discharge & $468(3.9)$ & $276(3.8)$ & $192(4.0)$ & 0.67 \\
\hline At 3-month & $990(8.2)$ & $606(8.4)$ & $384(8.0)$ & 0.44 \\
\hline At 6-month & $1270(10.6)$ & $774(10.7)$ & $496(10.3)$ & 0.49 \\
\hline At 12-mont & $1602(13.2)$ & $995(13.8)$ & $607(12.6)$ & 0.07 \\
\hline
\end{tabular}

Abbreviation: IQR Interquartile Range, TIA Transient Ischemic Attack, COPD Chronic obstructive pulmonary disease, mRS Modified Rankin Scale, EMS Emergency Medical System, SBP Systolic Blood Pressure, DBP Diastolic Blood Pressure, NIHSS National Institutes of Health Stroke Scale score, OCSP Oxfordshire Community Stroke Project.

Additional file 1: Figure S1]). The median age was 67 (IQR $57-75$ ) and $61.6 \%$ were male. The median length of hospital stay was 14 days (IQR: 10-20). The median admission NIHSS score was 5 (IQR: 2-9). The proportion of good functional outcome $(\mathrm{mRS} \leq 2)$ at discharge, 3-month, 6-month, and 1-year after AIS was 67.9\%, $66.5 \%, 66.9 \%$ and $66.9 \%$, respectively. The eligible patients were randomly divided into derivation $(60 \%$, $\mathrm{n}=7,215)$ and validation cohort $(40 \%, \mathrm{n}=4,811)$, which were matched with respect to baseline characteristics and overall rate of good functional outcome $(\mathrm{mRS} \leq 2)$ at different time points following AIS (Table 1).

\section{Derivation of the DFS-AIS}

The multivariate analysis for predictors of good functional outcome $(\mathrm{mRS} \leq 2)$ at discharge, 3-month, 6-month, and 1 -year after AIS is shown in Table 2. Age, gender, medical history of diabetes mellitus, stroke/TIA (for $\mathrm{mRS} \leq 2$ at 3-month, 6-month and 1-year after onset), current smoking (for $\mathrm{mRS} \leq 2$ at discharge) and atrial fibrillation (for $\mathrm{mRS} \leq 2$ at 3 -month, 6-month and 1-year after onset), pre-stroke dependence, pre-stroke statins using (for $\mathrm{mRS} \leq 2$ at 3-month, 6-month and 1-year after onset), admission NIHSS score, admission blood glucose were identified as independent predictors of functional outcome at different time points after AIS. The tolerance of covariates in the final multivariable model ranged 0.80-0.99 and the VIF 1.01-1.21 (Table 2). The probability of good functional outcome $(\mathrm{mRS} \leq 2)$ at discharge, 3-month, 6-month, and 1-year after AIS can be estimated for an individual patient by a web-based calculator (Figure 1) (www.dfs-ais.com).

\section{Validation of the DFS-AIS}

Discrimination of the DFS-AIS with regard to good functional outcome $(\mathrm{mRS} \leq 2)$ at discharge, 3-month, 6-month, and 1-year after AIS is shown in Additional file 1: Figure S2 (an additional figure shows this in more detail [see Additional file 1: Figure S2]). Similar good discrimination was found in both the derivation (AUROC range: $0.842-0.845$ ) and validation cohort (AUROC range: 0.837-0.841). The Hosmer-Lemeshow tests of the DFSAIS for good functional outcome at discharge, 3-month, 6-month, and 1-year after AIS were significant in the validation cohort (all $\mathrm{P}<0.001$ ). However, a graph of observed versus predicted likelihood of good functional outcome at discharge, 3-month, 6-month, and 1-year after AIS showed a close correlation between observed and predicted risk in both the derivation and validation cohorts (all $r=0.99, \mathrm{P}<0.001$ ) (an additional figure shows this in more detail [see Additional file 1: Figure S3]), which indicated excellent calibration.

\section{Sensitivity analysis}

We completed pre-specified subgroup analyses by age, gender, time intervals from stroke onset to arrival, OCSP subtype and thrombolysis. Similar good discrimination was seen in these subgroups (AUROC range: 0.773-0.917), especially for those patients with TACI subtype (AUROC range: 0.855-0.917) (Table 3).

\section{Comparison of AIS risk models}

Discrimination of the DFS-AIS and 8 existing AIS models for good functional outcome $(\mathrm{mRS} \leq 2)$ and mortality at discharge, 3-month, 6-month and 1-year after AIS in the validation cohort $(\mathrm{n}=4,811)$ is shown in Figure 2 (additional tables show this in more detail [see Additional file 1: Table S2 and S3]). For good functional outcome prediction, although all tested models showed acceptable discrimination (AUROC $\geq 0.70$ ), the DFS-AIS demonstrated significantly higher AUROC than other models (all $\mathrm{P}<0.0001$ ). Meanwhile, the DFS-AIS had the highest maximum Youden Index and associated sensitivity, specificity, PPV and NPV (an additional table shows this in more detail [see Additional file 1: Table S2]). Similar results were found for predicting mortality at discharge, 3-month, 6-month, and 1-year after AIS (Figure 2 and Additional file 1: Table S3). When the comparison was performed in the overall cohort $(\mathrm{n}=12,026)$, similar results was found (see Additional file 1: Table S4 and S5). 
Table 2 Multivariable analysis

\begin{tabular}{|c|c|c|c|c|c|c|c|c|c|c|c|c|}
\hline \multirow[t]{2}{*}{ Predictors } & \multicolumn{3}{|c|}{ Discharge $\mathrm{mRS} \leq 2^{*}$} & \multicolumn{3}{|c|}{ 3-month mRS $\leq 2^{*}$} & \multicolumn{3}{|c|}{ 6-month $\mathrm{mRS} \leq 2^{*}$} & \multicolumn{3}{|c|}{1 -year $\mathrm{mRS} \leq 2^{*}$} \\
\hline & B-coefficient & OR (95\% C.I.) & $\mathbf{P}$ & B-coefficient & OR (95\% C.I.) & $P$ & B-coefficient & OR (95\% C.I.) & $\mathbf{P}$ & ß-coefficient & OR (95\% C.I.) & $\mathbf{P}$ \\
\hline Intercept & 2.855 & & & 4.010 & & & 4.355 & & & 3.892 & & \\
\hline Age (per 1 year increase) & -0.02 & $0.98(0.97-0.98)$ & $<0.0001$ & -0.043 & $0.96(0.95-0.96)$ & $<0.0001$ & -0.050 & $0.95(0.95-0.96)$ & $<0.0001$ & -0.058 & $0.94(0.94-0.95)$ & $<0.0001$ \\
\hline Gender (male) & 0.295 & $1.34(1.19-1.52)$ & $<0.0001$ & 0.234 & $1.26(1.14-1.40)$ & $<0.0001$ & 0.189 & $1.21(1.09-1.34)$ & $<0.0001$ & 0.274 & $1.32(1.18-1.47)$ & $<0.0001$ \\
\hline \multicolumn{13}{|l|}{ Risk factors } \\
\hline Diabetes mellitus (No) & 0.204 & $1.23(1.08-1.39)$ & 0.001 & 0.289 & $1.34(1.17-1.52)$ & $<0.0001$ & 0.296 & $1.34(1.18-1.53)$ & $<0.0001$ & 0.239 & $1.27(1.11-1.45)$ & $<0.0001$ \\
\hline Stroke/TIA (No) & $\ldots$ & $\cdots$ & $\cdots$ & 0.278 & $1.32(1.18-1.49)$ & $<0.0001$ & 0.316 & $1.37(1.22-1.55)$ & $<0.0001$ & 0.371 & $1.45(1.30-1.62)$ & $<0.0001$ \\
\hline Current smoking (No) & 0.157 & $1.17(1.03-1.33)$ & 0.01 & $\ldots$ & $\ldots$ & & & & & & & $\ldots$ \\
\hline Atrial fibrillation (No) & $\ldots$ & $\ldots$ & $\cdots$ & 0.218 & $1.24(1.02-1.52)$ & 0.03 & 0.230 & $1.26(1.03-1.53)$ & 0.03 & 0.303 & $1.35(1.11-1.65)$ & 0.003 \\
\hline $\begin{array}{l}\text { Pre-stroke independence } \\
(\mathrm{mRS} \leq 2)\end{array}$ & 0.549 & $1.73(1.47-2.04)$ & $<0.0001$ & 0.588 & $1.80(1.51-2.15)$ & $<0.0001$ & 0.672 & $1.96(1.65-2.33)$ & $<0.0001$ & 0.634 & $1.89(1.58-2.22)$ & $<0.0001$ \\
\hline pre-stroke statins using (Yes) & $\cdots$ & $\cdots$ & $\cdots$ & 0.198 & $1.22(1.10-1.35)$ & $<0.0001$ & 0.213 & $1.24(1.12-1.37)$ & $<0.0001$ & 0.263 & $1.30(1.17-1.44)$ & $<0.0001$ \\
\hline $\begin{array}{l}\text { Admission NIHSS score } \\
\text { (per } 1 \text { increase) }\end{array}$ & -0.197 & $0.82(0.81-0.83)$ & $<0.0001$ & -0.211 & $0.81(0.80-0.82)$ & $<0.0001$ & -0.196 & $0.82(0.81-0.83)$ & $<0.0001$ & -0.185 & $0.83(0.82-0.84)$ & $<0.0001$ \\
\hline $\begin{array}{l}\text { Admission BG (per } 1 \mathrm{mmol} / \mathrm{L} \\
\text { increase) }\end{array}$ & -0.044 & $0.96(0.94-0.98)$ & $<0.0001$ & -0.043 & $0.95(0.93-0.97)$ & $<0.0001$ & -0.054 & $0.95(0.93-0.97)$ & $<0.0001$ & -0.061 & $0.94(0.92-0.96)$ & $<0.0001$ \\
\hline
\end{tabular}

Multivariable logistic regression adjusted for age, gender, stroke risk factors, pre-existing comorbidities, pre-stroke dependence, pre-admission medications (anticoagulant with warfarin, anti-platelet medication, and statins), transport model to hospital, time from onset to hospital arrival, admission systolic and diastolic blood pressure, NIHSS score, OCSP subtypes, and admission blood glucose.

*Probability of good functional outcome $(m R S \leq 2)$ is calculated by $P=e^{Y} /\left(1+e^{Y}\right)$, where $Y=a+b_{1} X_{1}+b_{2} X_{2}+\ldots .+b_{i} X_{i}$;

Abbreviation: OR Odds Ratio, C.I. Confidence Interval, VIF Variance Inflation Factor, TIA Transient Ischemic Attack, mRS Modified Rankin Scale, NIHSS National Institutes of Health Stroke Scale score, BG Blood glucose. 


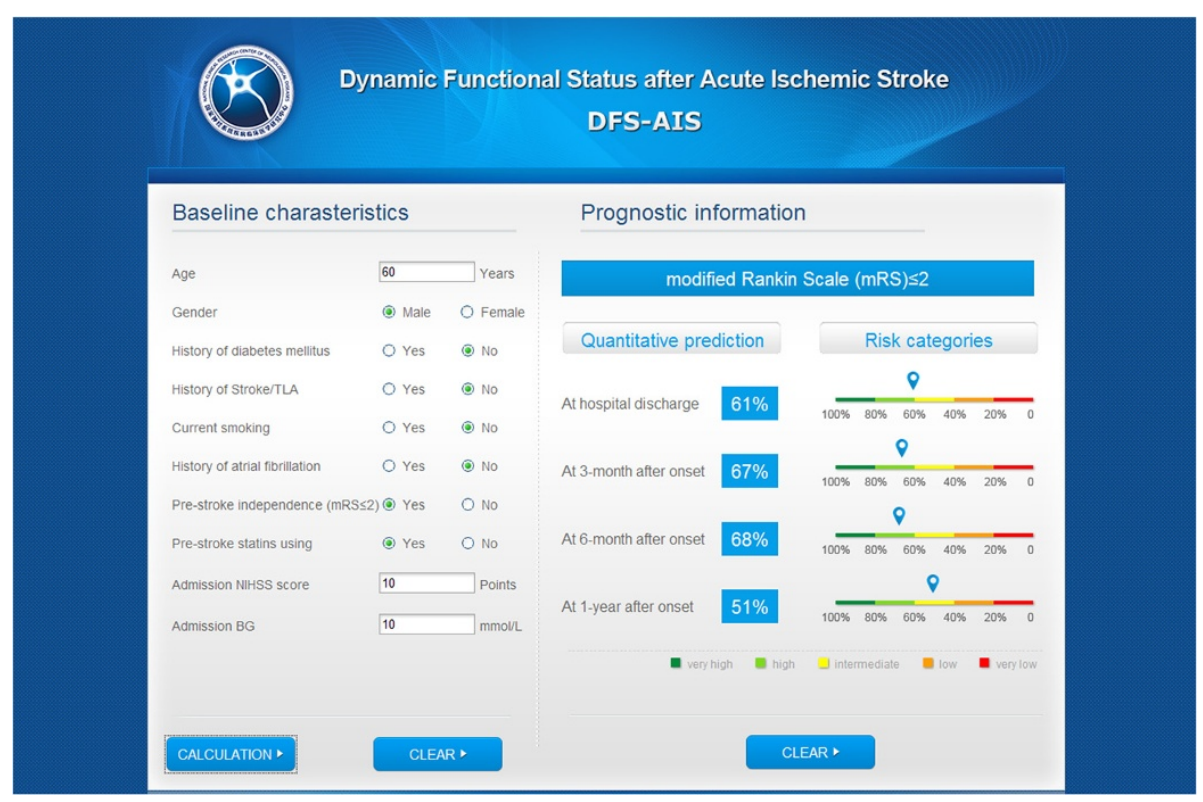

Figure 1 The web-based calculator for the DFS-AIS.

\section{Discussion}

In the study, we developed and validated a risk model to predict functional outcome at multiple time points (discharge, 3-month, 6-month, and 1-year) after AIS by using information routinely available at presentation.
A web-based risk model (the DFS-AIS) was developed based on the set of independent predictors of each outcome measures and showed good discrimination and calibration in large derivation and validation cohorts. When compared to 8 existing models, the DFS-AIS

Table 3 Sensitivity analysis of the DFS-AIS in the overall cohort $(n=12,026)$

\begin{tabular}{|c|c|c|c|c|c|c|c|c|}
\hline & \multicolumn{2}{|c|}{ Discharge $\mathrm{mRS} \leq 2$} & \multicolumn{2}{|c|}{ 3-month mRS $\leq 2$} & \multicolumn{2}{|c|}{ 6-month $\mathrm{mRS} \leq 2$} & \multicolumn{2}{|c|}{1 -year mRS $\leq 2$} \\
\hline & AUROC & 95\% C.I. & AUROC & 95\% C.I. & AUROC & 95\% C.I. & AUROC & 95\% C.I. \\
\hline \multicolumn{9}{|l|}{ Age } \\
\hline$\leq 59$ & 0.845 & $0.829-0.861$ & 0.826 & $0.808-0.844$ & 0.820 & $0.801-0.839$ & 0.813 & $0.793-0.833$ \\
\hline$\geq 60$ & 0.830 & $0.820-0.839$ & 0.836 & $0.826-0.846$ & 0.830 & $0.820-0.840$ & 0.827 & $0.817-0.837$ \\
\hline \multicolumn{9}{|l|}{ Gender } \\
\hline Male & 0.834 & $0.823-0.845$ & 0.832 & $0.821-0.843$ & 0.834 & $0.823-0.845$ & 0.834 & $0.823-0.845$ \\
\hline Female & 0.843 & $0.830-0.856$ & 0.849 & $0.837-0.861$ & 0.846 & $0.834-0.858$ & 0.845 & $0.833-0.857$ \\
\hline \multicolumn{9}{|l|}{ Time from onset to arrival (hours) } \\
\hline$\leq 3$ & 0.829 & $0.808-0.850$ & 0.846 & $0.825-0.867$ & 0.847 & $0.826-0.868$ & 0.857 & $0.837-0.877$ \\
\hline $3-6$ & 0.838 & $0.808-0.868$ & 0.826 & $0.796-0.856$ & 0.836 & $0.807-0.865$ & 0.834 & $0.805-0.863$ \\
\hline $6-24$ & 0.834 & $0.818-0.850$ & 0.845 & $0.830-0.860$ & 0.842 & $0.827-0.857$ & 0.848 & $0.833-0.862$ \\
\hline$\geq 24$ & 0.845 & $0.833-0.856$ & 0.841 & $0.829-0.853$ & 0.839 & $0.828-0.850$ & 0.831 & 0.819-0.842 \\
\hline \multicolumn{9}{|l|}{ OCSP subtype } \\
\hline Lacunar infarction (LACI) & 0.809 & $0.785-0.833$ & 0.819 & $0.796-0.842$ & 0.829 & $0.807-0.851$ & 0.816 & $0.794-0.838$ \\
\hline Partial anterior circulation infarct (PACl) & 0.827 & $0.816-0.838$ & 0.821 & $0.810-0.832$ & 0.822 & $0.811-0.833$ & 0.826 & $0.815-0.837$ \\
\hline Total anterior circulation infarct (TACl) & 0.855 & $0.830-0.880$ & 0.917 & $0.900-0.936$ & 0.912 & $0.892-0.932$ & 0.905 & $0.885-0.925$ \\
\hline Posterior circulation infarct (POCl) & 0.853 & $0.833-0.873$ & 0.846 & $0.826-0.866$ & 0.840 & $0.820-0.860$ & 0.845 & $0.826-0.846$ \\
\hline Intravenous or intra-arterial thrombolysis & 0.773 & $0.724-0.822$ & 0.785 & $0.735-0.833$ & 0.779 & $0.733-0.825$ & 0.781 & $0.730-0.832$ \\
\hline
\end{tabular}

Abbreviation: mRS Modified Rankin Scale, AUROC Area Under the Receiver Operating Characteristic Curve, C.I. Confidence Interval, OCSP Oxfordshire Community Stroke Project. 


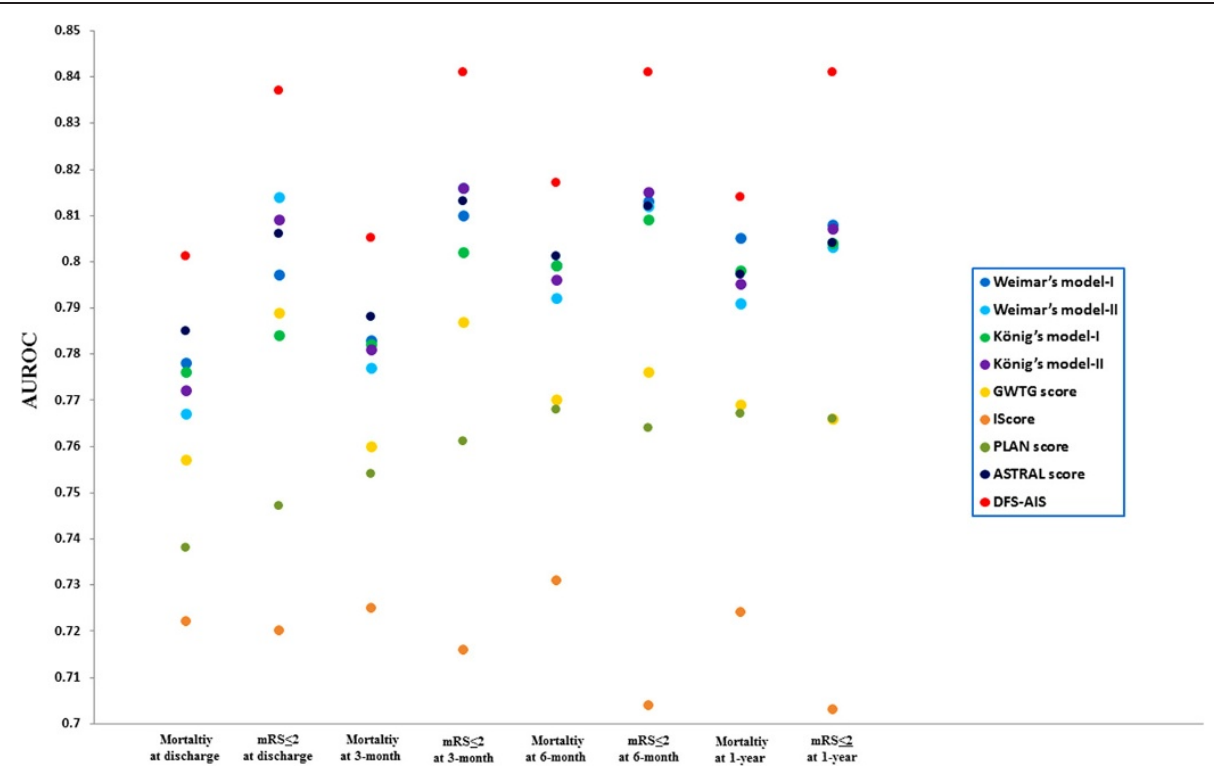

Figure 2 Comparative evaluation of the DFS-AIS and 8 existing AIS models. Figure 2 showed discrimination of the DFS-AIS and 8 existing AIS models with regard to good functional outcome $(\mathrm{mRS} \leq 2)$ and mortality at discharge, 3-month, 6-month, and 1-year after AIS in the validation cohort $(n=4,811)$. The DFS-AIS consistently showed significant better discrimination than compared models with regard to both good functional outcome $(m R S \leq 2)$ and mortality at discharge, 3-month, 6-month, and 1-year after AIS (all P<0.0001).

showed significantly better discrimination with regard to both functional outcome and mortality at discharge, 3-month, 6-month, and 1-year after AIS.

In order to preserve clinical utility of the model for decision-making during acute hospitalization and postdischarge, we used only patient characteristics available at presentation. We chose not to include variables related to in-hospital and postdischarge management, such as intravenous recombinant tissue plasminogen activator (rt-PA) [22], rehabilitation, and persistence of evidence-based secondary prevention medications [27-29], despite the fact that these therapies could influence functional status following AIS. This model therefor predicts the expected functional status following AIS at presentation.

For a clinical prognostic rule to become effective and widely used, it must be reliable, accurate and practicable [3]. For reliability, the DFS-AIS was developed based on large derivation and validation cohorts, which included consecutive patients of AIS from a nationwide stroke registry, was outside of clinical trials, and was more reflective of real-world clinical practice; In addition, by sensitivity analysis, the DFS-AIS demonstrated to be effective for patients with various clinical characteristics, such as different age, gender, time intervals from stroke onset to hospital arrival, and OCSP subtype. For accuracy, the DFS-AIS was proven to be accurate in risk-stratification and outcome prediction for both functional outcome and mortality at multiple time points after AIS. For practicability, the DFS-AIS consists of factors that are readily available at hospital presentation. In addition, by a web-based and user-friendly calculator, users could obtain prognostic information at multiple time points following AIS without doing complex calculation.

To the best of our knowledge, we are the first to systematically compare AIS risk models in a single population. During the past decades, a multitude of AIS predictive models have been developed. Although each of these prognostic rules has inherent strengths and limitations, none of them is widely accepted and used in routine clinical practice or clinical trials $[3,19]$. With many AIS prognostic rules available, identification of the most accurate and reliable model(s) would be of great value to patients, family members, clinicians, and researchers. In this study, we found that the DFS-AIS consistently demonstrated significant better discrimination than 8 compared models for both functional outcome and mortality at discharge, 3-month, 6-month, and 1-year after AIS (Figure 2, Table S1, and S2). Though promising, one should exercise caution when interpreting the results: first, the study populations for derivation and validation of these AIS risk models are different. The clinical characteristics of our study population were different from those of western cohorts used to develop compared models $[13,15,17,18]$, such as with younger age of AIS onset, higher proportion of male gender, less severity of neurological deficit on admission, and lower rate of mortality at different time points following AIS. It is not our primary aim to compare difference of these cohorts and it is hard to explain the potential reasons due to sorts of difference in study design and population. 
Second, there might be complex genetic, social, culture, economic factors as well as regional management philosophies and preferences that are difficult to account for when prognostic models are developed or applied to a distinct population. Finally, the intended outcome (functional outcome vs. mortality), outcome assessment methods (Barthel Index vs. mRS), and timing of followup (in-hospital vs. 30-day vs. 3-month vs. 6-month vs. 1-year after AIS) are different for these AIS models. One might argue it is not fair to directly compare these models for functional outcome and mortality at various time points after AIS as that they were not designed in that way. However, our intention was not to show limitation of these models beyond the context under which they are developed, but to provide evidence on which model is more appropriate for what kind of outcome (functional outcome vs. mortality) and at what specific time points (discharge, 3-month, 6-month, and 1-year) after AIS.

By offering prediction of functional outcome at multiple time points following AIS, rather than only mortality, the DFS-AIS can be useful for patients, family members, clinicians and researchers, whose primary concern is not the likelihood of survival, but rather the likelihood of survival with recovery of function. This is also important since different clinical care contexts or clinical research may focus on short-term or long-term functional outcome after AIS differently. Meanwhile, the DFS-AIS could be used to monitor the performance of hospitals or stroke units in stroke care. Those hospitals or stroke units which deviate in outcome from the expected outcome (e.g. worse outcome) need to be monitored in detail to identify the potential targets for improvement. In addition, like other prognostic tools, the DFS-AIS may help in randomized clinical trials to stratify study population, in nonrandomized clinical trials to control for case-mix variation, and in controlled clinical trials to select suitable patients and to reduce required sample size $[19,30,31]$.

Our study has limitations that deserve comment. First, like all observational studies, we cannot rule out the possibility that additional variable (unmeasured confounders) might have some impact on functional status after stroke, such as intravenous rt-PA, neuroimaging characteristics, rehabilitation, and persistence of evidence-based secondary prevention medications. However, given our emphasis on early prediction using information at presentation to predict dynamic functional outcome after AIS, the DFS-AIS might be helpful to guide subsequent in-hospital and post-discharge management. Second, our study included only hospitalized stroke patients and those patients died in emergency department or treated in outpatient clinics were not included. Meanwhile, like most registries, our registry required informed consent and selection bias was inevitable [32]. Third, due to that the Medical Research Council score, volume of ischemia on diffusion-weighted imaging, Charlson comorbidity index, Consortium for the Investigation of Vascular Impairment of Cognition scale score, body temperature, and oxygen administration were not routinely collected in the CNSR, several other AIS models cannot be externally validated in the study $[4-6,8,11,14,16]$. Finally, the DFS-AIS is not externally validated in the study. Meanwhile, both the derivation and validation cohorts originated from Asian population, and therefore, the score needed to be further validated in non-Asian populations.

\section{Conclusion}

The DFS-AIS is a valid clinical model for predicting functional outcome at discharge, 3-month, 6-month, and 1-year after AIS. For clinical practice, the DFS-AIS can be useful for patients, family members, and clinicians by offering prediction of functional outcome at multiple time points following AIS. For clinical research, the DFS-AIS may help in randomized clinical trials to stratify study population, in nonrandomized clinical trials to control for case-mix variation, and in controlled clinical trials to select suitable patients and to reduce required sample size. Further validation of the DFS-AIS in different populations is required.

\section{Additional file}

\begin{abstract}
Additional 1: Table S1. Patient characteristics; Table S2. Discrimination of the DFS-AIS and 8 existing models for functional outcome after AIS in the validation cohort ( $n=4,811)$; Table S3. Discrimination of the DFS-AIS and 8 existing models for mortality after AIS in the validation cohort $(n=4,811)$; Table S4. Discrimination of the DFS-AIS and 8 existing models for functional outcome after AIS in the overall cohort $(n=12,026)$; Table S5. showed comparison of discrimination of the DFS-AIS and 8 existing models for functional outcome at discharge, 3-month, 6-month and 1-year after AIS in the overall cohort. Table S6. showed comparison of discrimination of the DFS-AIS and 8 existing models for mortality at discharge, 3-month, 6-month and 1-year after AIS in the overall cohort. Figure S1. Patient flowchart: Figure S2. Discrimination of the DFS-AIS for dynamic functional outcome after AIS. Figure S3. Plot of observed versus predicted likelihood of good functional outcome at multiple time points after AIS; Additional file 1: The CNSR investigators; Additional file 1: Institutional Review Board within the CNSR network.
\end{abstract}

\section{Abbreviations}

AIS: Acute ischemic stroke; AUROC: Area under the receiver operating characteristic curve; C.I.: Confidence interval; CNSR: China national stroke registry; COPD: Chronic obstructive pulmonary disease; CRF: Case report form; CT: Computerized tomography; DBP: Diastolic blood pressure; DFSAIS: Dynamic functional status after acute ischemic stroke; EMS: Emergency medical system; GIB: Gastrointestinal bleeding; IQR: Interquartile range; LACI: Lacunar infarction; MRI: Magnetic resonance imaging; mRS: Modified rankin scale; NIHSS: National Institutes of health stroke scale score; NPV: Negative predictive value; OCSP: Oxfordshire community stroke project; OR: Odds ratio; PACl: Partial anterior circulation infarct; POCl: Posterior circulation infarct; PPV: Positive predict value; SBP: Systolic blood pressure; SE: Standard error; TACI: Total anterior circulation infarct; TIA: Transient ischemic attack; VIF: Inflation factor; WBC: White cell count. 


\section{Competing interests}

The authors declare that they have no competing interests.

\section{Authors' contributions}

RJ and YoW conceived of the study, participated in its design, and drafted the manuscript. HS and RJ carried out statistical analysis. RJ, WD, HS, YP, PW $G L, Y i W, H L, X Z$, YoW participated in analysis or interpretation of data, and revised the manuscript for important intellectual content. All authors read and approved the final manuscript.

\section{Acknowledgement}

The CNSR is funded by the Ministry of Science and Technology (Dr. Yongjun Wang, 2006BA101A11) and the Ministry of Health of the People's Republic of China (Dr. Yongjun Wang, 2009CB521905) (in study design and data collection). This work was supported by the Nova Program of Beijing Science and Technology Commission (Dr. Ruijun Ji, 2008B30) (in statistical analysis, interpretation of data, writing of the manuscript and in the decision to submit the manuscript for publication).

\section{Author details}

${ }^{1}$ Tiantan Comprehensive Stroke Center, Beijing Tiantan Hospital, Capital Medical University, No. 6 Tiantanxili, Beijing 100050, Dongcheng District, China. ${ }^{2}$ China National Clinical Research Center for Neurological Diseases (NCRC-ND), Beijing, China. ${ }^{3}$ Department of Statistics and Operation Research, University of North Carolina, Chapel Hill, NC, USA.

Received: 2 July 2014 Accepted: 31 October 2014

Published online: 25 November 2014

\section{References}

1. Go AS, Mozaffarian D, Roger VL, Benjamin EJ, Berry JD, Blaha MJ, Dai S, Ford ES, Fox CS, Franco S, Fullerton HJ, Gillespie C, Hailpern SM, Heit JA, Howard VJ, Huffman MD, Judd SE, Kissela BM, Kittner SJ, Lackland DT, Lichtman JH, Lisabeth LD, Mackey RH, Magid DJ, Marcus GM, Marelli A, Matchar DB, McGuire DK, Mohler ER 3rd, Moy CS et al.: Executive summary: heart disease and stroke statistics-2014 update: a report from the American Heart Association. Circulation 2014, 129(3):399-410.

2. Gillespie $C D$, Wigington $C$, Hong Y, Centers for Disease C, Prevention: Coronary heart disease and stroke deaths - United States, 2009. Morb Mortal Wkly Rep Surveill Summ 2013, 62(Suppl 3):157-160.

3. Counsell C, Dennis M: Systematic review of prognostic models in patients with acute stroke. Cerebrovasc Dis 2001, 12(3):159-170.

4. Baird AE, Dambrosia J, Janket S, Eichbaum Q, Chaves C, Silver B, Barber PA, Parsons M, Darby D, Davis S, Caplan LR, Edelman RE, Warach S: A three-item scale for the early prediction of stroke recovery. Lancet 2001, 357(9274):2095-2099

5. Counsell C, Dennis M, McDowall M, Warlow C: Predicting outcome after acute and subacute stroke: development and validation of new prognostic models. Stroke 2002, 33(4):1041-1047.

6. Weimar C, Ziegler A, Konig IR, Diener HC: Predicting functional outcome and survival after acute ischemic stroke. J Neurol 2002, 249(7):888-895.

7. Johnston KC, Connors AF Jr, Wagner DP, Haley EC Jr: Predicting outcome in ischemic stroke: external validation of predictive risk models. Stroke 2003, 34(1):200-202.

8. Wang Y, Lim LL, Heller RF, Fisher J, Levi CR: A prediction model of 1-year mortality for acute ischemic stroke patients. Arch Phys Med Rehabil 2003, 84(7):1006-1011.

9. Weimar C, Konig IR, Kraywinkel K, Ziegler A, Diener HC, German Stroke Study C: Age and National Institutes of Health Stroke Scale Score within 6 hours after onset are accurate predictors of outcome after cerebral ischemia: development and external validation of prognostic models. Stroke 2004, 35(1):158-162.

10. Birkner MD, Kalantri S, Solao V, Badam P, Joshi R, Goel A, Pai M, Hubbard AE: Creating diagnostic scores using data-adaptive regression: an application to prediction of 30-day mortality among stroke victims in a rural hospital in India. Ther Clin Risk Manag 2007, 3(3):475-484.

11. Solberg OG, Dahl M, Mowinckel P, Stavem K: Derivation and validation of a simple risk score for predicting 1-year mortality in stroke. J Neurol 2007, 254(10):1376-1383.

12. Konig IR, Ziegler A, Bluhmki E, Hacke W, Bath PM, Sacco RL, Diener HC, Weimar C, Virtual International Stroke Trials Archive I: Predicting long-term outcome after acute ischemic stroke: a simple index works in patients from controlled clinical trials. Stroke 2008, 39(6):1821-1826.

13. Smith EE, Shobha N, Dai D, Olson DM, Reeves MJ, Saver JL, Hernandez AF, Peterson ED, Fonarow GC, Schwamm LH: Risk score for in-hospital ischemic stroke mortality derived and validated within the get with the Guidelines-Stroke Program. Circulation 2010, 122(15):1496-1504.

14. Reid JM, Gubitz GJ, Dai D, Kydd D, Eskes G, Reidy Y, Christian C, Counsell CE, Dennis M, Phillips SJ: Predicting functional outcome after stroke by modelling baseline clinical and CT variables. Age Ageing 2010, 39(3):360-366.

15. Saposnik G, Kapral MK, Liu Y, Hall R, O’Donnell M, Raptis S, Tu JV, Mamdani M, Austin PC, Investigators of the Registry of the Canadian Stroke Network Stroke Outcomes Research Canada (SORCan) Working Group: IScore: a risk score to predict death early after hospitalization for an acute ischemic stroke. Circulation 2011, 123(7):739-749.

16. Muscari A, Puddu GM, Santoro N, Zoli M: A simple scoring system for outcome prediction of ischemic stroke. Acta Neurol Scand 2011, 124(5):334-342.

17. Ntaios G, Faouzi M, Ferrari J, Lang W, Vemmos K, Michel P: An integer-based score to predict functional outcome in acute ischemic stroke: the ASTRAL score. Neurology 2012, 78(24):1916-1922.

18. O'Donnell MJ, Fang J, D'Uva C, Saposnik G, Gould L, McGrath E, Kapral MK, Investigators of the Registry of the Canadian Stroke N: The PLAN score: a bedside prediction rule for death and severe disability following acute ischemic stroke. Arch Intern Med 2012, 172(20):1548-1556.

19. Kasner SE: Clinical interpretation and use of stroke scales. Lancet Neurol 2006, 5(7):603-612.

20. Wang Y, Cui L, Ji X, Dong Q, Zeng J, Wang Y, Zhou Y, Zhao X, Wang C, Liu L, Nguyen-Huynh MN, Claiborne Johnston S, Wong L, Li H, China National Stroke Registry Investigators: The China National Stroke Registry for patients with acute cerebrovascular events: design, rationale, and baseline patient characteristics. Int J Stroke 2011, 6(4):355-361.

21. Ringleb PA, Bousser MG, Ford G, Bath P, Brainin M, Caso V, Cervera A, Chamorro A, Cordonnier C, Csiba L, Davalos A, Diener HC, Ferro J, Hacke W, Hennerici M, Kaste M, Langhorne P, Lees K, Leys D, Lodder J, Markus HS, Mas JL, Mattle HP, Muir K, Norrving B, Obach V, Paolucci S, Ringelstein EB, Schellinger PD, Sivenius J et al.: European Stroke Organisation (ESO) Executive Committee; ESO Writing Committee: Guidelines for management of ischaemic stroke and transient ischaemic attack. Cerebrovasc Dis 2008, 25(5):457-507.

22. Jauch EC, Saver JL, Adams HP Jr, Bruno A, Connors JJ, Demaerschalk BM, Khatri P, McMullan PW Jr, Qureshi Al, Rosenfield K, Scott PA, Summers DR, Wang DZ, Wintermark M, Yonas H, American Heart Association Stroke Council; Council on Cardiovascular Nursing; Council on Peripheral Vascular Disease; Council on Clinical Cardiology: Guidelines for the early management of patients with acute ischemic stroke: a guideline for healthcare professionals from the American Heart Association/American Stroke Association. Stroke 2013, 44(3):870-947.

23. Bamford J, Sandercock P, Dennis M, Burn J, Warlow C: Classification and natural history of clinically identifiable subtypes of cerebral infarction. Lancet 1991, 337(8756):1521-1526.

24. Cook NR: Use and misuse of the receiver operating characteristic curve in risk prediction. Circulation 2007, 115(7):928-935.

25. Kramer AA, Zimmerman JE: Assessing the calibration of mortality benchmarks in critical care: the Hosmer-Lemeshow test revisited. Crit Care Med 2007, 35(9):2052-2056.

26. DeLong ER, DeLong DM, Clarke-Pearson DL: Comparing the areas under two or more correlated receiver operating characteristic curves: a nonparametric approach. Biometrics 1988, 44(3):837-845.

27. Bushnell CD, Zimmer LO, Pan W, Olson DM, Zhao X, Meteleva T, Schwamm L, Ovbiagele B, Williams L, Labresh KA, Peterson ED: Persistence with stroke prevention medications 3 months after hospitalization. Arch Neurol 2010, 67(12):1456-1463.

28. Bushnell CD, Olson DM, Zhao X, Pan W, Zimmer LO, Goldstein LB, Alberts MJ, Fagan SC, Fonarow GC, Johnston SC, Kidwell C, Labresh KA, Ovbiagele B, Schwamm L, Peterson ED, AVAlL Investigators: Secondary preventive medication persistence and adherence 1 year after stroke. Neurology 2011, 77(12):1182-1190.

29. Ji R, Liu G, Shen H, Wang Y, Li H, Peterson E, Wang Y: Persistence of secondary prevention medications after acute ischemic stroke or transient ischemic attack in Chinese population: data from China National Stroke Registry. Neurol Res 2013, 35(1):29-36. 
30. Young FB, Lees KR, Weir CJ, Committee GITS Investigators: Improving trial power through use of prognosis-adjusted end points. Stroke 2005, 36(3):597-601.

31. Lees KR, Hankey GJ, Hacke W: Design of future acute-stroke treatment trials. Lancet Neurol 2003, 2(1):54-61.

32. Tu JV, Willison DJ, Silver FL, Fang J, Richards JA, Laupacis A, Kapral MK, Investigators in the Registry of the Canadian Stroke N: Impracticability of informed consent in the Registry of the Canadian Stroke Network. N Engl J Med 2004, 350(14):1414-1421.

doi:10.1186/s12883-014-0214-z

Cite this article as: Ji et al: Web-based tool for dynamic functional

outcome after acute ischemic stroke and comparison with existing models. BMC Neurology 2014 14:214.

\section{Submit your next manuscript to BioMed Central and take full advantage of:}

- Convenient online submission

- Thorough peer review

- No space constraints or color figure charges

- Immediate publication on acceptance

- Inclusion in PubMed, CAS, Scopus and Google Scholar

- Research which is freely available for redistribution 